\title{
Zukünftige Therapieansätze der Depressionsbehandlung
}

Die therapeutischen Optionen bei psychischen Erkrankungen wurden in den letzten Dekaden vielfältig entwickelt. Gleichwohl existieren unverändert teilweise bisher nicht adressierte, therapeutische Bedürfnisse, die auch zukünftig im Fokus der Forschungsanstrengungen stehen werden.

Die Depression repräsentiert eine der häufigsten psychischen Erkrankung. Die durchschnittliche Lebenszeitprävalenz der Depression liegt in Deutschland bei 9,9\% und bei $3 \%$ für die 12-Monatsprävalenz (Bromet et al., 2011). Die WHO schätzt, dass im Jahr 2030 Depressionen zu den führenden volkswirtschaftlichen Krankheitsbelastungen weltweit gehören werden (Mathers et al., 2006). Depressionen sind beispielsweise verbunden mit einem hohen Risiko gegenüber einer Chronifizierung bzw. einer Wiedererkrankung. Ferner können Depressionen negative Auswirkungen auf die Entwicklung von Begleiterkrankungen haben (Angst et al., 2002).

Trotz der jahrelangen Entwicklung von Antidepressiva existieren weiterhin unmet needs in der Therapie. Hierzu zählen neben den Forderungen nach neuen und besseren Diagnosekriterien (Demyttenaere et al., 2001; Kennedy, 2008) auch neue Behandlungsziele (Mc Knight et al., 2009). Hieraus resultierende neue Behandlungsziele sind primär die Verbesserung der Verträglichkeit und der Adhärenz; aber auch die Verminderung der Non-Response-Rate, die Reduktion der Wirklatenz sowie der Interaktionen von Arzneimitteln. In einem Delphi-Panel zur Therapiepräferenz deutscher Ärzte in der antidepressiven Therapie wurden Parameter identifiziert, die für die Behandlung der Patienten relevant sind (Gründer et al., 2016b). Die Dimension Wirksamkeit wurde als besonders bedeutsam erachtet, wobei die Parameter Response, Remission und Recovery als besonders wichtig bezeichnet wurden. Die Dimension Lebensqualität, und hier besonders die Bewältigung des häuslichen Alltags und die psychische Leistungsfähigkeit, wurde im zweiten Rang als bedeutsam erachtet. An dritter Stelle wurde die Verträglichkeit und Sicherheit der Therapie benannt.
Unter Berücksichtigung dieser Anforderungen und des heterogenen Krankheitsbildes der Depression ist es nicht wahrscheinlich, dass ein Medikament mit einem singulären Wirkmechanismus diese Aufgaben erfüllen kann. Die zukünftige medikamentöse Therapie der Depression muss alternative Wirkmechanismen zu den heute verbreiteten anbieten. Beispielweise kann dies durch eine Kombination mehrerer pharmakologischer Wirkmechanismen erfolgen. Basis kann in diesem Fall die Kombination von Antidepressiva mit verschiedenen Wirkmechanismen oder die Augmentation sein. Kontrollierte klinische Studien existieren nur für wenige Kombinationen von Antidepressiva. Dieses Vorgehen gewinnt jedoch in der ärztlichen Praxis zunehmend an Bedeutung. Als Alternative zur Kombination verschiedener Medikamente können Substanzen mit synergistischen Wirkmechanismen in einem Molekül erachtet werden.

Die Neuentwicklung eines Pharmakons bis zur Zulassung beansprucht heute bis zu 20 Jahre. Die Wahrscheinlichkeit, dass eine Substanz tatsächlich den Markt erreicht, beträgt dabei weniger als 10\%. In Deutschland existiert zudem seit 2011 eine zusätzliche Hürde für Arzneimittel in Form des AMNOG (Arzneimittelmarktneuordnungsgesetz). Zur Behandlung psychischer Störungen wurden bisher vier neu zugelassene Arzneimittel einer Nutzenbewertung gemäß AMNOG unterzogen. Für keines hat bisher der G-BA (Gemeinsamer Bundesausschuss) einen Zusatznutzen gegenüber der zweckmäßigen Vergleichstherapie anerkannt (Gründer, 2016). Zukünftig kann der Zugang deutscher Patienten zu neuen Medikamenten im Bereich Psychiatrie deutlich erschwert sein (Hamer und Meyer-Lindenberg, 2016).

Die gezielte Entwicklung eines Wirkstoffes erfordert unter anderem die Kenntnis des zu beeinflussenden Stoffwechselprozesses einschließlich des an dem Prozess beteiligten Zielmoleküls. Darüber hinaus ist das Wissen über die molekularen Wechselwirkungen zwischen Wirkstoff und Wirkort unabdingbar. Die Depression ist charakterisiert durch eine Vielzahl von Sympto- men, die in verschiedenster Art und Weise während einer depressiven Episode auftreten können. Hierbei basieren die verschiedenen Symptome der depressiven Episode nicht immer auf den gleichen neurobiologischen Mechanismen. Dies gilt zum einen für unterschiedliche Patienten, aber auch beim individuellen Patienten können in unterschiedlichen Erkrankungsperioden unterschiedliche pathophysiologische Prozesse den Symptomen zugrunde liegen.

Bei einer phänomenologisch heterogenen, multifaktoriell bedingten Erkrankung wie der Depression werden gegenwärtig primär selektive Arzneimittel zur Therapie eingesetzt. Der Trend geht jedoch zu einer Pharmakotherapie, die auf mehrere pharmakologische Ziele einwirkt. Bei den Strategien zur Entdeckung zukünftiger neuer Antidepressiva können zwei grundsätzliche Ansätze unterschieden werden. In der hypothesengeleiteten Forschung werden Wirkstoffe entwickelt, die sich am pathophysiologischen Modell der Depression orientieren. Es werden gegenwärtig zahlreiche pathophysiologische Modelle der Depression diskutiert, die zukünftig als Basis einer optimierten Therapie dienen können. Beim hypothesenfreien Forschungsansatz werden Biomarker erforscht, die die Entscheidungsfindung bei der Auswahl einer therapeutischen Intervention verbessern bzw. optimieren können, z. B. ob ein Arzneimittel beim individuellen Patienten zu einem positiven klinischen Ergebnis führt. Hierdurch können Patienten, die sich aufgrund ihrer Diagnose oder des Punktwertes auf einer Schweregradskala von anderen nicht unterscheiden, differenziert werden. Hierdurch kann eine Patienten-orientiertere Therapie ermöglicht werden.

In den letzten Jahren wurden nunmehr bevorzugt Substanzen mit pharmakologischer Wirkung an mehreren Neurotransmittersystemen entwickelt. Beispielhaft können hier Wirkstoffe mit neuen Wirkmechanismen aufgeführt werden wie Vilazodon und Vortioxetin, die unmet needs in der antidepressiven Wirksamkeit adressieren (Wang et al., 2015). Vilazodon repräsentiert einen Serotonin-Reuptake Inhibi- 
tor mit zusätzlicher agonistischer Wirkung auf den 5-HT 1 a-Rezeptor (Hughes et al., 2005). Hingegen wirkt Vortioxetin zusätzlich als Antagonist auf $5-\mathrm{HT}_{3}, 5-\mathrm{HT}_{1 \mathrm{~d}}$ und $5-\mathrm{HT}_{7}$ sowie als Partialagonist von $5-\mathrm{HT}_{1 \mathrm{~b}}$ (Dubovsky, 2014). Auf Basis der besonderen Bedeutung von Serotonin, Noradrenalin und Dopamin in der Pathophysiologie der Depression wurde eine Vielzahl von TRI (Triple Reuptake Inhibitoren) entwickelt, wobei Daten aus der klinischen Forschung zum Teil widersprüchliche $\mathrm{Er}$ gebnisse liefern. In der Zeitspanne 2006 bis 2012 wurden 146 Patente zu TRI publiziert; Beispiele sind Amitifadine, Lu AA42202, RG-7166 (Shao et al., 2014).

Ein schneller Wirkeintritt ist in der antidepressiven Therapie ein therapeutischer Bedarf, der primär durch Ketamin adressiert werden könnte (Überblick: Wang et al., 2015). Ketamin repräsentiert einen glutaminergen Modulator und NMDA-Rezeptoragonisten, der intensiv untersucht wurde (Rot et al., 2012). Seine Wirkung wurde in ersten klinischen Studien untersucht; eine schnelle antidepressive Wirksamkeit von Ketamin wurde dokumentiert (Rot et al., 2012; Krystal et al., 2013).

Weitere Targets können durch Neuropeptide beschrieben werden, die als Neurotransmitter bzw. Co-Transmitter fungieren und somit die Aktivität der monoaminergen Transmitter und die HPA-Achse (HPA: Hypothalamus-Pituitary Axis) modulieren. So wurde für NK1-Rezeptor-Antagonisten wie Aprepitant (MK-869) ein antidepressiver Effekt dokumentiert (Kramer, 2004); klinische Studien konnten die Wirksamkeit jedoch noch nicht verifizieren (Keller et al., 2006). Weitere Neuropeptide befinden sich in der präklinischen Forschung. Als weitere Targets mit antidepressiver Wirkung werden gegenwärtig unter anderem die Opiat-Rezeptoren, Sigma-Rezeptoren oder Purin-Rezeptoren untersucht.

Neben der Entwicklung neuer Wirkstoffe wird die Polypharmazie in der Behandlung der Depression angewendet, wobei nur wenige positive klinische Studien als Evi- denz zur Verfügung stehen. Ein ermutigendes Zeichen ist, dass jüngst ein Biomarker (miR-1202) identifiziert wurde, der für die Vorhersage der Wahrscheinlichkeit einer Response unter Citalopram verwendet werden kann (Lopez et al., 2014).

Zukünftige antidepressive Wirkstoffe sollten basierend auf der Pathophysiologie der Depression, d.h. auf Basis der neuronalen Netzwerke und unter Berücksichtigung der Interaktionen zwischen Transmittersystemen entwickelt werden.

\section{Interessenkonflikte}

MF ist Mitarbeiter der Lundbeck GmbH.

\section{Autor}

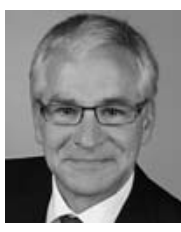

Michael Friede

Lundbeck GmbH, Hamburg

\section{Korrespondenzadresse}

\section{Dr. Michael Friede}

Head of Medical Business Area Central Lundbeck $\mathrm{GmbH}$

Ericusspitze 2

20457 Hamburg

lumf@lundbeck.com

\section{Literatur}

[1] Angst F, Stassen HH, Clayton PJ et al. Mortality of patients with mood disorders: followup over 34-38 years. J Affect Disorders 2002; 68: $167-181$

[2] Bromet E et al. Cross-national epidemiology of DSM-IV major depressive episode. BMC Med 2011; 9: 90

[3] Demyttenaere $\mathrm{K}$ et al. Compliance with antidepressants in a primary care setting. 1: Beyond lack of efficacy and adverse events. J Clin Psychiatry 2001; 62 (Suppl. 22): 30-33

[4] Dubovsky S. Pharmacokinetic evaluation of vortioxetine for the treatment of major depressive disorder: efficacy across symptoms and severity of depression. Int Clin Psychopharmacol 2014; 29: 759-766
[5] Gründer G. Bedeutung des Arzneimittelmarktneuordnungsgesetzes für die Psychopharmakotherapie. Der Nervenarzt 2016; 87: $356-366$

[6] Gründer $G$ et al. Präferenz von Zielparametern bei der Behandlung der Depression - Ergebnisse einer Delphi-Erhebung. Der Nervenarzt 2016; doi doi:10.1007/s00115016-0180-3

[7] Hamer H, Meyer-Lindenberg A. Auswirkungen des Arzneimittelmarktneuordnungsgesetzes (AMNOG) auf die neurologische und psychiatrische Versorgungsqualität. Der Nervenarzt 2016; 87: 351-352

[8] Hughes Z. et al. Neurochemical evaluation of the novel 5-HT1a receptor partial agonist/serotonin reuptake inhibitor, vilazodone. Eur J Pharmacol 2005; 10: 49-57

[9] Kennedy S. Core symptoms of major depressive disorders: relevance to diagnosis and treatment. Dialogues Clin Neurosci 2008; 10: $271-277$

[10] Keller M. et al. Lack of efficacy of the substance $\mathrm{p}$ (neurokinin 1 receptor) antagonist aprepitant in the treatment of major depressive disorder. Biol Psychiatry 2006; 59: 216-223

[11] Kramer $\mathrm{M}$ et al. Demonstration of the efficacy and safety of a novel substance $p$ (NK1) receptor antagonist in major depression. Neuropsychopharmacol 2005; 29: 385-302

[12] Krystal J et al. Rapid-acting glutamatergic antidepressants: The path to ketamine and beyond. Biol Psychiatry 2013; 73: 1133 1141

[13] Lopez J et al. miR-1202 is a primate-specific and brain-enriched microRNA involved in major depression and antidepressant treatment. Nature Medicine 2014; 20: 764-768

[14] Mathers C, Loncar D. Projections of global mortality and burden of disease from 2002 to 2030. PLoS 2006; 3: e442

[15] Mc Knight P, Kashdan T. The importance of functional impairment to mental health outcomes: a case for reassessing our goals in depression treatment research. Clin Psychol rev 2009; 29: $243-259$

[16] Rot M et al. Ketamine for depression: Where do we go from here? Biol Psychiatry 2012; 72: $537-547$

[17] Shao L. Triple reuptake inhibitors: a patent review (2006-2012). Expert Opin Ther Pat 2014; 24: $131-154$

[18] Wang $S$ et al. Criticisms of drugs in early development for the treatment of depression: What can be improved? Expert Opin Investig Drugs 2015; 24: $445-453$ 